\title{
Nuclear Medicine Clinical Practice in the United States During the COVID-19 Era and Beyond
}

\author{
Gail A. McFarland, CNMT, RT (CT) ${ }^{1}$ and Sara G. Johnson, CNMT, NCT ${ }^{2}$ \\ ${ }^{I}$ Nuclear Medicine Technology Program Bellevue College, Bellevue, Washington; and ${ }^{2}$ Nuclear Medicine Service, VA San Diego \\ Healthcare System, San Diego, California
}

\begin{abstract}
Traditionally, practice in nuclear medicine has involved strong emphasis on radiation safety principles. Nuclear medicine technologists (NMTs) focus on practices that keep patients, the public, and the technologist safe from potentially harmful effects of unnecessary radiation exposure using concepts of time, distance, and shielding as well as ALARA (As low as reasonably achievable) principles. The current COVID-19 pandemic has brought to light the need to apply focus on infection prevention in practice and update knowledge and procedures on such measures. In this article, the authors outline the need for NMTs to develop practices and values focused on infection prevention measures.
\end{abstract}

Key Words: safety; nuclear medicine; COVID-19

J Nucl Med Technol 2020; 48:218-226

DOI: 10.2967/jnmt.120.253245

\section{$\mathbf{S}$} edented infectious disease situation unlike anything experienced in modern times (1). The disease, coronavirus disease 2019 (COVID-19), caused by the virus severe acute respiratory syndrome coronavirus 2 (SARS-CoV-2) has spread widely to pandemic proportions and threatens to overwhelm health-care systems throughout the world. At the height of the pandemic, the Centers for Disease Control and Prevention (CDC) (2) recommended cancelling elective procedures, which constitutes most of the work that is done in nuclear medicine departments. The disease is observed to spread easily with human-to-human contact, putting health-care workers and patients alike at risk (2). It is impossible to determine who might be infectious given the high prevalence of asymptomatic and presymptomatic individuals. Therefore, nuclear medicine departments in many locations have limited procedures to urgent and emergency cases, and are functioning far below capacity.

Received Jul. 8, 2019; revision accepted Jul. 17, 2020.

For correspondence and reprints contact: Gail A. McFarland, Bellevue

College, 3000 Landerholm Circle SE, Bellevue, WA 98007-6484.

E-mail: gail.mcfarland@bellevuecollege.edu

Published online Jul. 24, 2020.

COPYRIGHT (c) 2020 by the Society of Nuclear Medicine and Molecular Imaging.
Coronaviruses are RNA viruses that primarily affect birds and mammals. In humans, they can be mild but can also cause more serious respiratory tract infections. These viruses have previously manifested as severe acute respiratory syndrome (SARS), Middle East respiratory syndrome (MERS), and now COVID-19. These viruses have emerged since the 21st century and have caused respiratory disease outbreak. However, each has unique features. SARS and MERS have much higher case fatality rates than COVID-19, but COVID-19 is much more infectious. Nevertheless, COVID-19 overall deaths are much higher than either SARS or MERS at 130,133 in the United States (3).

The World Health Organization (WHO) issued a global alert about SARS in March of 2003 with recommendations that only essential travel to affected countries be permitted (Hong Kong, Toronto, and several areas of mainland China and Taiwan). The WHO went on to declare SARS an epidemic in July 2003. To date the total number of deaths of SARS has been 812 worldwide, with 73 of those deaths in the United States. MERS was confirmed in April 2012 and is a zoonotic virus, meaning that the primary source of infection is from animals to humans, with the dromedary camels as the most common route of infection. MERS is seldom transmitted among people, but it most often occurs among family members or in health-care settings. The total number of cases of MERS worldwide is 2,519, with 2 cases in the United States (3).

The SARS-CoV-2 virus is known to be highly transmissible, spreading easily by human-to-human transmission $(4,5)$. This may be due, in part, to the high susceptibility of human T-lymphocytes to SARS-CoV-2, compared with other coronaviruses (6). Most commonly, the mode of spread is via droplets from the respiratory tract and direct contact between people. Creation of airborne virus-containing particles that travel on air currents is known as "aerosol" generation and is a third means for spread of the infection $(7,8)$. With airborne spread, it is thought that desiccation of droplets leads to airborne virus-containing nuclei that potentially spread widely. This phenomenon has been observed experimentally with SARS, MERS, and influenza (4). Stool samples have also been found to contain SARS-CoV-2 in individuals with negative respiratory tests (5). Other possible routes include fecal-oral and placental spread, although the latter appears to 
be much less likely given that few clear cases of maternalfetal spread have been documented. During the SARS outbreak of 2002-2003, it was documented that cases of SARS spread through faulty plumbing systems in a building in Hong Kong (4). It is also important to consider that there is a high prevalence of infected persons who are asymptomatic, mildly symptomatic, and presymptomatic. Given the vast potential for spread of SARS-CoV-2 and emergence of new viral diseases, all staff, patients, and visitors in nuclear medicine facilities are considered to present potential risks to those who are present.

Reduced capacity in nuclear medicine cannot continue indefinitely. Therefore, it will be important for nuclear medicine personnel to adopt procedures that facilitate safe practice using the highest level of infection prevention principles. Patients will need reassurance that they will be safe during nuclear medicine visits (9). Continued safety will come through updated infection prevention policies based on scientific data applied to practice.

In the past, safety measures specific to controlling infections have used a wide range of philosophies, myths, and policy interpretations according to technologist and management personal preferences and perceptions (10). In the months before the COVID-19 crisis, many techs continued to use outdated personal practices or practices based on preferences and incorrect assumptions. Given that COVID19 appears to be a long-term concern, nuclear medicine technologists (NMTs) and others find themselves faced with profound social and cultural changes with regard to their professional and personal practices (11).

\section{DEMAND TO OPEN NONURGENT AND ROUTINE HEALTH SERVICES}

For financial reasons as well as providing for routine and less urgent care needs for patients in communities, there is a demand for opening up health-care systems as soon as feasible (9). Furloughed workers need to be back to work in routine health-care roles and to relieve others who are overwhelmed with critically ill patients. This includes nuclear medicine operations. However, patients with nonurgent care needs also need to know that they are safe from the threat of contracting COVID-19 during care (9). Therefore, it is imperative that health-care workers demonstrate the highest levels of safety and continued quality.

Nuclear medicine practice is centered on routine and less urgent care in most cases. Nuclear medicine examinations and therapies are designed with physiologic principles in mind, and therefore have unique and specific requirements and issues for delivery (12). Certain examinations such as ventilation/perfusion (V/Q) lung scans present high risk for spread of SARS-CoV-2 to NMTs and others who might enter the nuclear medicine department. Specific hygiene rules along with technical and nontechnical considerations must be followed to minimize disease risk for all. Updated infection prevention information and practice from the CDC and WHO should to be tailored specifically to nuclear medicine departments so as to minimize risk in the current pandemic situation and for infectious disease outbreaks that are anticipated to arise in the future (12).

\section{PARALLELS WITH RADIATION SAFETY PRECAUTIONS AND OTHER PROTECTIVE PRINCIPLES}

NMTs have been applying radiation safety principles almost since the inception of nuclear medicine technology and as a profession MTs use the ALARA principle (As low as reasonably achievable) (13). This means that NMTs minimize time handling radioactive patients and sources, keep a safe distance to reduce radiation intensity, and use shielding for radioactive sources whenever feasible. Similar principles involving time, distance, and shielding can be applied to infection prevention measures (14). Specifically, workers reduce the risk of contracting an infectious disease by shielding themselves with personal protective equipment (PPE), maintaining a 3- to 6-ft buffer between themselves and potentially infectious patients, and limiting the time spent with these patients (2).

Nuclear medicine personnel, physicians, technologists, and scientists have traditionally put high value on protecting self, patients, and the public from unnecessary radiation risks. For safety during the current pandemic situation and to protect future health, it is imperative that NMTs put the same value on infection prevention with regard to nuclear medicine practice. As nuclear medicine professionals work to ensure continued radiation safety focus, comparable emphasis should be put on averting infectious disease risk.

\section{NECESSITY FOR DEVELOPMENT OF NUCLEAR MEDICINE-SPECIFIC INFECTION PREVENTION MEASURES}

The uniqueness of nuclear medicine examinations necessitates development of specific infection prevention procedures (12). NMTs perform diverse job responsibilities based on physiologic and functional examinations that include an assortment of imaging, laboratory, pharmacy, patient care, and housekeeping tasks. NMTs fit the criteria to be classified in multiple health-care worker specialties and will need to follow PPE recommendations for each $(15,16)$. Safety measures pertain to laboratory biosafety, aerosol concerns, equipment and environmental hygiene, and most importantly providing safe patient care (16). From a clinical perspective, supervisors are discovering that staff NMTs do not fully comprehend reasoning and procedures behind use of some types of PPEs. Additionally, educators are hearing anecdotes from students and practicing NMTs such as, "Gloves only protect me. They do not help the patient" and "Using hand sanitizer is only a policy given by administration, we don't really use it." However, technologists and students now see the manifestations of COVID-19 disease spreading through communities and health systems and must be willing to change their viewpoints for the common good.

NMTs have previously faced the need to change their clinical practices to meet the demands of unknown infectious 
agents, specifically the HIV and the advanced HIV infection known as AIDS (acquired immunodeficiency virus) in the 1980s (17). Before that time NMTs, like many health-care workers, used gloves and other PPE sporadically. But NMTs adjusted their clinical practices to routinely using PPE, especially gloves. NMTs had to learn to palpate and identify veins with gloves and devices such as vein lights. NMTs were quick to adjust and respond to the HIV and AIDS crisis and, as such, were able to continue practicing with minimal disruption of care to patients (17).

\section{PLANNING FOR PERSONAL HYGIENE MEASURES AND PPE IN NUCLEAR MEDICINE PRACTICE}

No infection prevention measure can entirely eliminate transmission of infectious agents, but risk is substantially lower when they are used properly and consistently (16). Health-care workers including NMTs are at higher risk compared with the public due to the high stress and fatigue that comes with working with patients during a pandemic situation, and from direct exposure to infected patients who have high viral loads compared with what is encountered outside of the health-care system. Education, clear intent, and planning are essential for consistent use of safety measures (5). It is for the greater good of all that NMTs adhere to the following safe practices:

\section{Hand Cleaning}

Before interacting with patients, the NMT must always perform hand hygiene (18). The most effective way to clean hands is with soap and water. Hand hygiene with alcoholbased hand rubs (60\%-95\% purity) works well if hands are not visibly soiled; regular washing at the sink is required if they are. Hygiene supplies including hand rubs, antimicrobial soap, paper towels, waste bins, and the like should be readily available in all locations that are adjacent to or involve patient care. NMTs should keep in mind the locations of sinks for situations that necessitate soap and water washing in place of hand rubs and make personal plans as to how and when they will use them (10). It can be expected that hand hygiene will need to be performed by each worker between 3 and 30 times per hour in a patient care setting to protect self and patient (19). Also, important to consider is the frequency of movement between imaging equipment, the patient, and the surfaces touched by human hands in the control room or desk areas around the imaging room. The many "hand touches" during nuclear medicine procedures presents many opportunities for hand hygiene.

\section{Glove Use}

NMTs entering patient care areas should do so with gloved hands $(2,16)$. Hands should be cleaned before putting on fresh gloves to avoid transfer of organisms. Gloves that are put on unwashed hands are considered contaminated. NMTs should expect to change gloves frequently, both before and after many types of patient care duties. The WHO campaign My Five Moments of Hand Hygiene specifies types of patient interactions that require hand cleaning and glove change including before and after touching a patient, handling bodily fluids, and touching patient surroundings (20).

\section{Face Coverings}

Currently, the CDC recommends that all health-care workers including NMTs cover the face with PPEs such as respirators and face masks (21). Cloth face coverings worn by the general public are not considered protective for those who work with patients, particularly those who have known or potential COVID-19 infections. Goggles or face masks should be considered to protect the eyes as well. Personal eyeglasses are not substitutes when eye protection is needed. Ventilation lung scans are aerosol-generating procedures and require wearing masks with higher protection capabilities such as N95 masks as well as full face shields (16).

\section{Isolation Gowns}

NMTs should wear isolation gowns when entering patient care areas (15). This includes nuclear medicine imaging rooms. With the high potential for SARS-CoV-2 spread, NMTs assigned to perform aerosol-generating procedures such as ventilation lung scans must be given priority when gowns are in short supply (21).

\section{Donning and Doffing Practices for Isolation Gear (Figs. 1 and 2)}

Before the COVID-19 pandemic, most NMTs put little thought into changing gloves except when contaminated with radioactivity or visibly soiled, and minimal consideration to proper use of items such as isolation gowns. Donning (putting on) isolation gear should be done in a manner that prevents self-contamination and maintains hygienic standards (15). Before donning, hand hygiene must be performed and plans should be devised based on which items will be laid out for use. The general order for putting on isolation gear starts with donning the isolation gown and is followed by putting on a mask or respirator, eye protection, and finally gloves (15). The NMT should also understand when PPE items will need to be exchanged, and where and how items will be disposed of when finished.

Doffing refers to removing gear when finished with patient care or a procedure (15). This should be done after the patient has been escorted away from the imaging room and before the NMT exits the room. The order for doffing includes first removing gloves because they are assumed to be contaminated, removing eyewear, and removing the isolation gown. If the hands become contaminated while doffing isolation gear, it will be necessary to perform hand cleaning. All contaminated items should be disposed of in an institution-approved waste bin. The face mask or respirator should be left on until after exiting the room.

\section{CLEANING AND HYGIENIC PRACTICE IN NUCLEAR MEDICINE DEPARTMENTS}

Cleaning in health-care settings has traditionally been focused on esthetic qualities rather than on tested scientific measures (22). Perceptions by the public and by hospital 


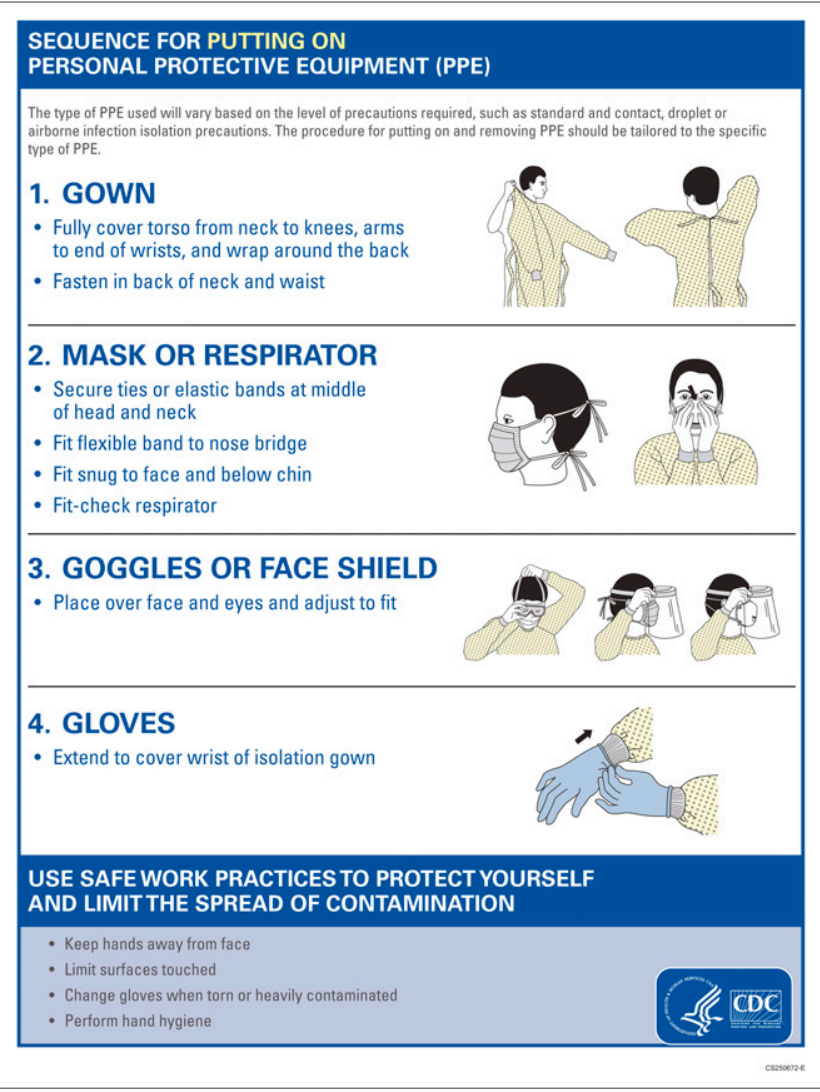

FIGURE 1. CDC sequence for putting on personal protective equipment (PPE). (Centers for Disease Control and Prevention; March 14, 2020. https://www.cdc.gov/hai/pdfs/ppe/ppesequence.pdf.)

personnel are that the appearance of "clean" constitutes good hygiene and sanitary practice. NMTs know intuitively based on surveys for radioactive contamination that appearance does not necessarily imply absence of contaminants. Area surveys with Geiger counters and survey meters demonstrate contamination with radioactivity, but similar assays to look for microbial contamination such as ATP bioluminescence tests are not in common use (23). Room cleaning in nuclear medicine departments (excluding radioactive wastes) has most often been done by housekeeping staff who are given low budgets and minimal training in hygienic techniques. However, effective control of pathogens in a hospital setting requires scientific cleaning procedures performed by persons with considerably more training than housekeepers have received in the past. Personnel who perform cleaning in health-care settings should be trained scientifically as technicians with an understanding of infectious agents, location of potentially contaminated surfaces, principles of disinfection and decontamination, and means to measure cleanliness (23). Recently, novel cleaning agents have been developed that are highly effective against resistant viruses and spore-forming bacteria. NMTs should understand and apply scientific cleaning principles themselves. With such knowledge, NMTs can ensure that nuclear medicine workspaces are safe for patients, visitors, other staff, and self.

\section{CHANGES IN DEPARTMENT MANAGEMENT}

\section{Patient Scheduling, Registration, Arrival, and Discharge}

In nuclear medicine departments, high throughput of procedures presents high risk for viral exposure and transmission. Prior scheduling practices often neglected to account for the infection prevention aspects of examinations, and during workdays NMTs routinely encountered subsequent patients before completing room cleaning, donning personal protection, or assessing the patient's situation. In postCOVID-19 times, changes in examination scheduling must be made to widen time slots to allow NMTs to perform these duties (12). Longer time slots and avoiding overbooking of examinations will also limit patient contact with others in waiting areas and ensure availability of adequate nuclear medicine personnel to attend to patients.

In addition, in order to provide adequate social distancing in waiting rooms, nuclear medicine patients must not bring additional family members to appointments. Social distancing has required most institutions to reduce the capacity of their waiting rooms, and as such it may not be

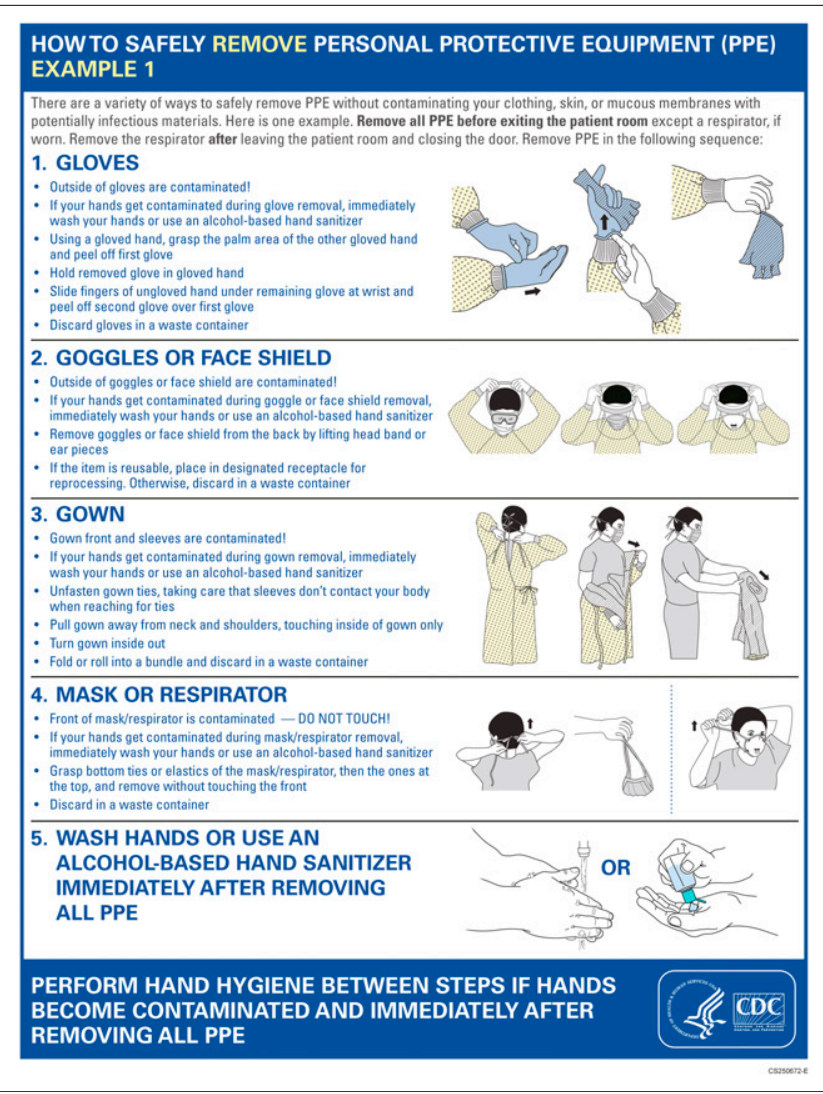

FIGURE 2. CDC sequence for safely removing personal protective equipment (PPE). (Centers for Disease Control and Prevention; March 14, 2020. https://www.cdc.gov/hai/pdfs/ ppe/ppe-sequence.pdf.) 
possible to for patients to arrive to their nuclear medicine appointments early. Many institutions are asking patients who arrive early to their appointments to wait in their cars until their appointment times; oftentimes a "virtual checkin" is used. The nuclear medicine department is notified that the patient has arrived and can thereby notify the patient when to come into the department. Because of these expanded appointment slots, this may require nuclear medicine departments to extend their clinic hours into the evening or into the weekend as well.

The registration process can increase time patients spend in close proximity to others in waiting rooms (12). Registration desks must be widely spaced with shields to ensure distance between admitting clerks and patients. Masks, hand-cleaning supplies, and instructions must be provided in waiting rooms also for patients and visitors. In addition, waiting room furniture and surfaces should be cleaned as necessary to ensure that any potential COVID-19 virus is removed. Visual measures that reinforce patient safety such as cleaning monitors and pens should be performed between each patient admission. This may require additional ancillary staff to monitor and clean waiting rooms. With heightened infection prevention needs, NMTs need to devote additional time for planning, setup, and donning personal protection before starting examinations as part of each examination workflow. Extra time is required also to ensure that each patient has sufficient PPE and assessment of symptoms. Neglecting any of these duties puts staff, patients, and visitors at risk for infection.

On arrival in the nuclear medicine department, inpatients with confirmed COVID-19 disease or patients under investigation (PUI) should be given masks to wear if that has not already been done (12). When a COVID-19 patient arrives at the department, they should be escorted directly to the imaging/procedure room and not stop in the waiting room or hallways. The NMT should be prepared with PPE before the arrival of the patient including an N95 mask, goggles or face shield, and isolation gown. In addition, some institutions may require head or shoe coverings. Donning and doffing procedures must be adhered to as described previously to ensure there is no possibility for contamination (15). The registration process can increase the time patients spend in close proximity to others in waiting rooms. To reduce the patient time in the department, there should be careful coordination of escort services to quickly return inpatients to their wards. In addition, the NMT should have all doses, flushes, and the ventilation aerosol unit or other equipment staged in the designated imaging room before the patient arrives.

After the procedure is completed and the patient has left the room, it should be closed to allow any residual particles to be cleared by the air system. In general, this would require 6-12 complete air exchanges (4). This process usually takes over an hour depending on air flow rates and building engineering requirements. After this time housekeeping should be called in to perform terminal cleaning (deep cleaning-as done with tuberculosis patients in many institutions). Once housekeeping has cleared the room, the technologist should thoroughly disinfect and wipe down the camera, camera collimators, imaging bed, hand controller, and computers. Housekeeping in general will not clean technical equipment. When cleaning is completed, the room can safety be put back into routine use. The process to turn over the imaging/procedure room after scanning SARSCoV-2-positive patients will add at least 90 min to any nuclear medicine study and this additional time will have to be accounted for in the clinical schedule.

\section{Resource Allocation}

Risk due to lack of human resources and ability to use sick time are legitimate concerns in nuclear medicine departments. Common complaints among NMTs are that there is a short supply of contingency staff available to fill in for absences due to budget decisions and technologist shortages. It is crucial that management devote sufficient resources to a float pool of NMTs to cover absences, and not punish those who need to be absent for personal illness or caring for sick family members. As mentioned above, escort services and registration staff are essential to safe department functions. Greater numbers of support staff need to be included in budgets.

Allocation of resources pertains also to PPE supplies. Media reports have brought up serious PPE shortages for nurses and physicians. However, NMTs encounter situations of risk comparable to nurses and physicians but are less visible to management. NMTs have sufficient reason to claim adequate supplies of items such as respirators, isolation gowns, gloves, and shoe protectors and should be given equitable access. Some state health regulations require that priority for PPE be given to workers with a likelihood of contact with infected persons, workers who have risk of spreading infections to vulnerable persons, and workers who need to perform aerosol-generating procedures (21). NMTs fit these criteria precisely in hospital settings, as noted with the risk of aerosolized particles exposure during lung ventilation studies (16). However, the procurement of PPE may be challenging as other areas in the facility (ICU, emergency room, surgery) are also competing for these limited resources. It is important that nuclear medicine management highlight that staff are dealing with the potential of "droplet contamination" during the performance of lung ventilation studies and MPI stress procedures. It is necessary to educate those responsible for supply chain management to the urgent need for PPE for nuclear medicine. In addition, NMTs should also be tested for COVID-19 virus based on individual institution policies.

Further, the CDC (24) states that health-care organizations should develop contingency plans for extra PPE supplies for ensuring operational safety for all in case of PPE shortages. Specific protocols should be developed to address the real needs of individual nuclear medicine departments. Nuclear medicine managers must consider usage 
rates to plan for daily patient loads and surge capacity. Surge capacity describes the need for additional PPE supplies during an outbreak or pandemic situation. The PPE supply stock should be based on the volume of nuclear medicine procedures (including after hours and on-call studies) that are able to be performed given the limitations due to COVID-19 restrictions. For example, a routine lung $\mathrm{V} / \mathrm{Q}$, which normally required $1 \mathrm{~h}$ to complete, now with COVID-19 requirements may take up to $3 \mathrm{~h}$.

\section{NUCLEAR MEDICINE ROOM AND EQUIPMENT DESIGN}

Because droplets expired by humans present a significant risk for viral transmission, adequate room airflow and ventilation are essential to imaging room safety (8). The current situation in health care is that patient care areas can carry significant risk when air flow is low. This risk, in turn, leads to inefficiencies because rooms must now be kept vacant until particle levels are estimated to have returned to safe levels. The registration process can increase time patients spend in close proximity to others in waiting rooms. Preferably, 6-2 room air exchanges should occur per hour $(4,18)$. If adequate mechanical (or natural) ventilation cannot be accomplished, air disinfection devices can be used (4). These devices include upper room germicidal UV (GUV) fixtures or less complex air disinfectors such as HEPA filters.

Ideally, ventilation should be an important component of all hospital areas, including nuclear medicine room design, with hospital remodels and new building projects. Room air contains organisms exhaled by patients so imaging rooms should be designed to cycle air outdoors, while bringing in fresh air from the outdoors. Heating, ventilation, and air conditioning systems (HVACs) that accomplish the ventilation process will also need to have filters to remove particles from exiting air and extract pollutants from air entering rooms (8). Imaging suites should also be updated to have sinks, hand-cleaning stations, and a private toilet for each room. Counter tops and other workspaces in imaging rooms can also be outfitted to have antiadhesive or antimicrobial surfaces that reduce organism proliferation and biofilm formation (23). Design updates can help to achieve reductions in both infectious agents and radioactive contamination.

In many nuclear medicine departments, there is noteworthy absence of portable equipment (12). In recent years, easily portable equipment that is lightweight and compact with battery longevity has been developed for use with isolation and ICU patients and should be used where practical. One example is the solid-state, flat panel mobile camera made by Digirad (25). These may be used for examinations such as V/Q, hepatobiliary, and gastrointestinal bleed scans at the patient's bedside. Memories of cumbersome old-style portable nuclear medicine equipment have caused some NMTs to be adamantly opposed to portable $\gamma$-cameras. However, radiologic technologists and ultrasonographers routinely move portable equipment to patient bedsides in emergency departments, surgical suites, and patient care wards. NMTs should also have the capacity for bedside imaging, which is useful to avoid bringing infectious or potentially infectious patients into nuclear medicine departments.

\section{NUCLEAR MEDICINE EXAMINATIONS}

\section{Patient Preparation}

As stated previously, patients who are SARS-CoV-2positive or PUI should be brought to the nuclear medicine department with an appropriate face mask. The NMT performing the examination should have had sufficient time and opportunity for planning, preparation, and examination setup. All efforts should be made to complete the examination efficiently and minimize time the patient must spend in the department. Infection prevention measures should be adhered to thoroughly as described above.

\section{Lung $\mathbf{V} / Q$ Imaging}

Early on in nuclear medicine practice, lung V/Q imaging was described as one of the most commonly performed studies (26). V/Q scans have declined in frequency as other diagnostic methods, notably the CT pulmonary angiogram, have become more popular. With the advent of SPECT and SPECT/CT, V/Q scanning has been significantly improved with a higher sensitivity and specificity compared with planar V/Q scanning (27). Nuclear medicine lung scans have continued to have a role in diagnosing pulmonary embolism (PE) and assessing other causes of chest pain and dyspnea. During the COVID-19 pandemic, it has been suggested by Zuckier et al. (28) that a shift to a diagnostic algorithm can reduce the number of required ventilation studies. However, this algorithm still identifies a population of patients who should go on to complete a V/Q scan with aerosol precautions.

The ventilation procedure can be performed with numerous agents including ${ }^{133} \mathrm{Xe},{ }^{99 \mathrm{~m}} \mathrm{Tc}$-diethylenetriaminepentaacetic acid (DTPA), ${ }^{81 \mathrm{~m}} \mathrm{Kr}$, or ${ }^{99 \mathrm{~m}} \mathrm{Tc}$-microparticles ( $99 \mathrm{~m} \mathrm{Tc}-T e c h n e g a s ;$ Cyclomedica) (29). Of these ventilation imaging agents, ${ }^{81 \mathrm{~m}} \mathrm{Kr}$ and ${ }^{99 \mathrm{~m}} \mathrm{Tc}-$ Technegas are not readily available in the United States. However, Cyclopharm Limited filed a 505(b) New Drug Application for Technegas with the U.S. Food and Drug Administration on March 30, 2020 (30). Before COVID19, ongoing clinical trials were active in the United States, which may make Technegas available in the next year in the United States.

Aerosolized procedures for lung scanning as with ${ }^{99 \mathrm{~m}} \mathrm{Tc}-$ DTPA present the highest risk for COVID-19 exposure to NMTs. The technologist must be in the room with the patient assisting during the ventilation procedure. In order to get an adequate count rate, the patient must inhale on the ventilation system for at least 1.5 to $3 \mathrm{~min}$. During this time, it is not uncommon to have some aerosolized particles escape from the patient's mouthpiece into the surrounding area and deposit contamination on the NMT and in the room environment. These particles are now understood to have potential COVID19 virus and, as such, are a hazard to technologists.

The noble gases, ${ }^{133} \mathrm{Xe}$ and ${ }^{81 \mathrm{~m}} \mathrm{Kr}$, for lung ventilation carry a smaller risk of exposure to COVID-19 for 2 reasons. Use of these gases requires the imaging room to have negative 
pressure, and the amount of time the patient has to be ventilated is usually less than a minute. Both of these factors will reduce the possibility of COVID-19 exposure to NMTs.

Finally, Technegas is certainly a promising agent that has been used extensively in Europe and Australia. This system uses an ultra-fine microaerosol, of graphite, called "bucky balls" (31). These bucky balls are delivered to the patient via a Technegas generator. $\mathrm{TcO}^{-}$is placed into an alcoholmoistened graphite crucible, which is heated to produce the bucky balls coated with ${ }^{99 \mathrm{~m}} \mathrm{Tc}$. The patient inhales via an attached breathing apparatus 2 or 3 times to get approximately $1.0 \mathrm{mCi}(37 \mathrm{MBq})$ into their lungs. Because only 2 or 3 puffs are necessary to get adequate images, this system greatly reduces potential COVID-19 exposure to technologist.

Regardless of the ventilation agent, every effort must be made to mitigate the risk of COVID-19 exposure with lung ventilation procedures. All patients who are to have lung ventilation imaging should have a COVID-19 test. For inpatients, COVID-19 testing is now a common procedure, but for outpatients this is more challenging. Patients who are having lung ventilation studies must have a COVID-19 test before their lung V/Q scan. This order will need to be placed by a nuclear medicine provider (2). At the present time, most facilities have initiated outpatient drive-up COVID-19 testing as a preprocedure requirement for aerosol-generating procedures. These testing centers are normally only open Monday through Friday. COVID-19 testing results normally take $1-2 \mathrm{~d}$ to be returned. As such, outpatient lung scans may have to be scheduled later in the work week to allow time for the patient to go to a testing center for the drive-up COVID-19 test and then to get the results back before the lung V/Q scan. In addition, patients should self-isolate between their COVID-19 test and their lung V/Q scan to reduce the chance of further COVID-19 exposure.

Once results are received, negative COVID-19 patients may proceed with their lung V/Q scan. Positive COVID-19 patients need to be referred to their primary care providers for management. Most facilities have a mechanism in place through their infection control service to trigger the appropriate notification of primary care physicians when a patient has a positive COVID-19 test. However, because the COVID-19 test was originally ordered by a nuclear medicine provider at the time the lung V/Q scan was scheduled, a policy must be put in place for the nuclear medicine service to ensure that primary care providers are notified of the positive COVID-19 test and determine whether the lung V/Q scan can be delayed until the patient is COVID-19-negative. Institutional policies will differ and are continually changing during this pandemic as new data becomes available, but minimally the nuclear medicine department must document that the patient's primary care physician was notified of the positive test and is agreeable to delaying or rescheduling the lung V/Q scan.

Another challenge that is being recognized with this pandemic for nuclear medicine departments is that scans are requested of patients who have previously been COVID-19-positive. Again, institutional policy will differ but in general the CDC recommendation is that a COVID19 patient should have negative results of a Food and Drug Administration Emergency Use Authorized COVID-19 molecular assay for detection of SARS-CoV-2 RNA from at least 2 consecutive respiratory specimens collected $\geq 24 \mathrm{~h}$ apart (total of 2 negative specimens) (2). However, if a patient must be ventilated who is known to have COVID19 or is a PUI, as in emergency cases, every precaution should be taken to ensure that there is no cross-contamination. NMTs can use the following principles of time, distance, and shielding for protection from COVID-19 exposure during ventilation scans: Time-the least amount of time with the patient the better. The number of people who come in contact with the patient should be limited, such that preferably only 1 technologist performs the scan; distance - the further away from the patient the technologist can be, the better. It has been noted that COVID-19 particles can travel up to $6 \mathrm{ft}$; shielding - the "barrier" is PPE and negative pressure rooms rather than lead. If possible, the patient should be ventilated in a room with negative pressure. If there are not rooms with negative pressure, then a room with a single camera that can be closed off after the procedure should be used.

\section{Myocardial Perfusion Imaging (MPI)}

MPI is a well-validated, noninvasive method to assist with the determination of diagnostic and prognostic information for patients with suspected or known coronary artery disease. Images are obtained during rest and then during myocardial hyperemia, induced by exercise or pharmacologic stress agent. Rest and stress images are compared to determine the coronary flow reserve and identify areas of compromised myocardial perfusion. Stress testing puts the technologist in close proximity with the patient for a prolonged period of time. The preferred method for inducing hyperemia is exercise, but pharmacologic stress is performed in more than $50 \%$ of patients $(32,33)$. Most pharmacologic stress MPI studies are performed with the vasodilators adenosine, dipyridamole, or regadenoson. Making use of vasodilators, particularly regadenoson, rather than treadmill stress tests shortens procedure time and reduces equipment contamination and exposure to NMTs, leading to reduced risk of viral transmission (12). The side effects most often seen from these agents are mild and include flushing, chest pain, dyspnea, dizziness, and nausea. Should the nausea progress to vomiting, the NMT has added risk for COVID-19 exposure (33). The technologist is the primary caregiver for the patient and will need to perform additional cleaning measures.

The NMT should also make extra notes of cardiac arrhythmias, low ejection fraction, and myocarditis (12). These issues are reported to be correlated with some cases of COVID-19 and should be relayed to the supervising nuclear medicine physician or cardiologist. 


\section{Therapies and Hybrid Imaging}

NMTs should keep in mind that cancer patients may have greater risk for contracting COVID-19, and for severe symptoms should they contract the disease (34). Susceptibility also depends on individual patients' conditions and comorbidities. Assessment with the oncology team should be made to determine the need for urgency for a given patient's therapeutic (and any related diagnostic) procedures. Procedures that are of lower value or are not urgent should be postponed until effects of the pandemic subside (35). However, asymptomatic patients undergoing PET/CT and SPECT/CT scans have been found to have COVID-19 from the chest CT results of these imaging scans (36). As such, NMTs must continue to use COVID-19 precautions to protect themselves and other from asymptotic carriers. Some patients, for example, those needing hyperthyroidism therapy, can often be managed temporarily with medication until an appropriate time for the radionuclide treatment can be arranged (12).

\section{Postexamination Procedures}

Thorough imaging room cleanup should occur after each patient leaves the imaging room. This includes disinfecting $\gamma$-camera gantries, imaging tables, computer keyboards and mouse, sphygmomanometer and blood pressure cuff, infusion pump, treadmill, chairs, and all surfaces that may have been touched by the patient or someone with potentially contaminated gloves (12). Terminal cleaning can be done by housekeeping staff according to policy, but ultimately NMTs are responsible for cleaning and handling imaging equipment and medical devices.

\section{CONCLUSION}

Due to the COVID-19 pandemic, the way we practice all medicine, including nuclear medicine, will change. The pandemic situation is changing nearly on a daily basis, with new information being learned and updated policies put in place. The COVID-19 pandemic has identified of infection prevention deficiencies during nuclear medicine procedures and practice. The process of using aerosolized ${ }^{99 \mathrm{~m}} \mathrm{Tc}$-DTPA particles for lung ventilation scans, performing myocardial perfusion stress testing, and potential encounters with bodily fluids are areas of concern for staff contamination. Inevitably, a new normal will emerge with respect to infection prevention practice in medicine. NMTs will need to practice with intentional and thoughtful planning and heightened focus on safety. Nuclear medicine as a profession is generally on the forefront of new practices and must be willing to permanently adopt these for the future. For the present, nuclear medicine as a profession will need to focus on sharing information and collectively updating practices. Like radiation exposure risk, infectious disease risk will never be zero for NMTs working in any capacity but should be kept as low as reasonably achievable through updated knowledge, procedures, practices, and structural changes.

\section{DISCLOSURE}

No potential conflict of interest relevant to this article was reported.

\section{REFERENCES}

1. Coccolini F, Sartelli M, Kluger Y, et al. COVID-19 the showdown for mass casualty preparedness and management: the Cassandra Syndrome. World J Emerg Surg. 2020;15:26.

2. Centers for Disease Control and Prevention. Interim infection prevention and control recommendations for patients with suspected or confirmed coronavirus disease 2019 (COVID-19) in healthcare settings: summary of changes to the guidance. May 18, 2020. CDC website. https://www.cdc.gov/coronavirus/2019ncov/hcp/dialysis.html. Accessed August 15, 2020.

3. How do SARS and MERS compare with COVID-19? Medical News Today website. https://www.medicalnewstoday.com/articles/how-do-sars-and-mers-comparewith-covid-19. Accessed July 29, 2020.

4. Nardell EA. Nathavitharama, RR. Airborne spread of SARS-CoV-2 and a potential role for air disinfection. JAMA. June 1, 2020 [Epub ahead of print].

5. Bulut C, Kato Y. Epidemiology of COVID-19. Turk J Med Sci. 2020;50:563-570.

6. Ahmadpoor D, Ahmadpoor P. How the COVID-19 overcomes the battle? An approach to virus structure. Iran J Kidney Dis. 2020;14:167-172.

7. Tran K, Cimon K, Severn M, et al. Aerosol generating procedures and risk of transmission of acute respiratory infections to healthcare workers: a systematic review. PLoS One. 2012;7:e35797.

8. Morawska L, Tang JW, Bahnfleth W. How can airborne transmission of COVID19 indoors be minimised? Environ Int. 2020;142:105832.

9. Asch DA. Opening hospitals to more patients during the COVID-19 pandemicmaking it safe and making it feel safe. JAMA Intern Med. May 27, 2020 [Epub ahead of print].

10. Larson E. Monitoring hand hygiene: meaningless, harmful, or helpful? Am J Infect Control. 2013;41:S42-S45.

11. Berwick D. Changes for the 'New Normal'. JAMA. May 4, 2020 [Epub ahead of print].

12. Ayan A, Kirac FS. Guide for nuclear medicine applications during the COVID19 Outbreak. Mol Imaging Radionucl Ther. 2020;29:49-58.

13. Centers for Disease Control and Prevention. Radiation safety: ALARA - As Low As Reasonably Achievable. December 7, 2015. CDC website. https://www.cdc.gov/ nceh/radiation/alara.html\#: :text=The\%20guiding\%20principle\%20of\%20radiation, should $\% 20$ try $\% 20$ to\%20avoid\%20it. Accessed July 29, 2020.

14. Mukherji A, Gupta T, Agarwal JP. Time, distance, shielding and ALARA; drawing similarities between measures for radiation protection and Coronavirus disease pandemic response. Indian J Cancer. 2020;57:221-223.

15. Centers for Disease Control and Prevention. Personal protective equipment: questions and answers, coronavirus disease 2019 (COVID-19). March 14, 2020. CDC website. https://www.cdc.gov/coronavirus/2019-ncov/hcp/respirator-use-faq. html\#Gloves. Accessed July 29, 2020

16. Ağalar C, Ozturk Engin D. Protective measures for COVID-19 for healthcare providers and laboratory personnel. Turk J Med Sci. 2020;50(SI-1):578-584.

17. History of HIV and AIDS overview: interactive timeline; October 10, 2010. Avert.org website. https://www.avert.org/professionals/history-hiv-aids/overview. Accessed July 29, 2020.

18. Centers for Disease Control and Prevention. Guidelines for environmental infection control in health-care facilities: recommendations of CDC and the Healthcare Infection Control Practices Advisory Committee (HICPAC). 2003. CDC website. https://espanol.cdc.gov/infectioncontrol/pdf/guidelines/environmental-guidelinesP.pdf. Accessed July 29, 2020

19. Sax H, Allegranzi B, Uckay I. My five moments for hand hygiene: a user-centred design approach to understand, train, monitor and report hand hygiene. $J$ Hosp Infect. 2007;67:9-21

20. World Health Organization (WHO). My 5 moments for hand hygiene. Infection prevention and control. WHO website. https://www.who.int/infection-prevention/ campaigns/clean-hands/5moments/en/. Accessed July 29, 2020.

21. Washington State Department of Health (DOH). Interim prioritization guidelines for allocation of personal protective equipment. April 29, 2020. DOH website. https://www. doh.wa.gov/Portals/1/Documents/1600/coronavirus/PPEPrioritizationofAllocation. pdf. Accessed July 29, 2020.

22. Dancer SJ. The role of environmental cleaning in the control of hospital-acquired infection. J Hosp Infect. 2009;73:378-385.

23. Dancer SJ. Controlling hospital-acquired infection: focus on the role of the environment and new technologies for decontamination. Clin Microbiol Rev. 2014;27:665-690. 
24. Centers for Disease Control and Prevention. Strategies to optimize the supply of PPE and equipment. May 18, 2020. CDC website. https:/www.cdc.gov/coronavirus/ 2019-ncov/hcp/ppe-strategy/index.html. Accessed July 29, 2020

25. Digirad Corporation. Cameras: Digirad's solid-state benefits. Digirad website. https://www.digirad.com/cameras/. Accessed July 29, 2020.

26. Wagner HN Jr, Sabiston DC Jr, McAfee JG. Diagnosis of massive pulmonary embolism in man by radioisotope scanning. N Engl J Med. 1964;271:377384.

27. Roach PJ, Schembri GP, Bailey DL. VQ Scanning using SPECT and SPECT/CT. J Nucl Med. 2013;54:1588-1596.

28. Zuckier LS, Moadel RM, Haramiti LB, Freeman LM. Diagnostic evaluation of pulmonary embolism during the COVID-19 pandemic. J Nucl Med. 2020;61: 630-631.

29. Parker JA, Coleman RE, Grady E, et al. SNM practice guidelines for lung scintigraphy 4.0. J Nucl Med Technol. 2012;40:57-65.

30. Cyclopharm News. https://investor.cyclopharm.com/site/PDF/1555_0/40PatientMilestoneAchievedForTechnegasUSFDATrial. Accessed August 20, 2020.
31. Leblanc M, Tessier M, Ollenberger G, O’Brien C. CANM guidelines for ventilation/perfusion (V/P SPECT) in pulmonary embolism. CANM website. https://canm-acmn.ca/resources/Documents/Guidelines_Resources/ MasterDocument_Final_Nov_21_incl-Exec-Sum_ver3_Dec.\%2012_.pdf. Accessed July 29,2020

32. Gonzalez JA, Beller GA. Choosing exercise or pharmacologic stress imaging, or exercise ECG test alone: how to decide. J Nucl Cardiol. 2017;24:555-557.

33. Johnson SG, Peters S. Advances in pharmacologic stress agents: focus on regadenoson. J Nucl Med Technol. 2010;38:163-171.

34. Chua MLK, Yu J, Xie C. Risk of COVID-19 in patients with cancer-reply. JAMA Oncology. July 2, 2020 [Epub ahead of print].

35. Gyawali B, Poudyal BS, Eisenhauer EA. Covid-19 pandemic - an opportunity to reduce and eliminate low-value practices in oncology? JAMA Oncol. July 2, 2020 [Epub ahead of print].

36. Albano D, Bertagna F, Bertolia M, et al. Incidental findings suggestive of COVID19 in asymptomatic patients undergoing nuclear medicine procedures in a high prevalence region. J Nucl Med. 2020;61:632-636. 